\title{
Real-time Measurements with Atmospheric Instruments at the Pierre Auger Observatory
}

\author{
Violet M. Harvey ${ }^{* a}$ for the Pierre Auger Collaboration ${ }^{\star b}$ \\ ${ }^{a}$ Department of Physics, University of Adelaide, Adelaide, S.A. 5005, Australia \\ ${ }^{b}$ Observatorio Pierre Auger, Av. San Martín Norte 304, 5613 Malargüe, Argentina \\ E-mail: auger_spokespersons@fnal.gov \\ Full author list: http://www.auger.org/archive/authors_icrc_2019.html
}

\begin{abstract}
The state of the atmosphere regarding the air density profile, the vertical distribution of aerosols, and the location of clouds is closely monitored at the Pierre Auger Observatory because the troposphere serves as a giant calorimeter for the air fluorescence technique. We present an overview of the atmospheric monitoring instruments at our disposal, with a focus on their capabilities for real-time measurements and their importance to extensive air shower reconstruction. We detail the improvement of our database of aerosol attenuation measurements that has been extended by two more years to the end of 2017 and fully recalculated with new software that brings a range of enhancements. We also address the importance of using hourly measurements of aerosol distributions for analyses rather than static models of average atmospheric conditions.
\end{abstract}

36th International Cosmic Ray Conference - ICRC2019

24 July - 1 August, 2019

Madison, Wisconsin, USA

\footnotetext{
* Speaker.

${ }^{\dagger}$ For collaboration list see PoS(ICRC2019)1177.
} 


\section{Introduction}

The Pierre Auger Observatory [1], located at approximately $1400 \mathrm{~m}$ above sea level (ASL) in the Mendoza province of Argentina, is the world's largest detector of cosmic rays. It operates as a hybrid detector, with a $3000 \mathrm{~km}^{2}$ surface detector array (SD) composed of over 1600 waterCherenkov stations overlooked by a fluorescence detector (FD) composed of 27 telescopes divided among four sites. The FD detects fluorescence light from nitrogen molecules in the atmosphere excited by the passage of the secondary particles produced in an extensive air shower, while the SD detects the secondary particles at ground.

The air fluorescence technique for observing cosmic ray events and reconstructing the primary particle energy, the depth of shower maximum, and the arrival direction is nearly calorimetric because the quantity of isotropically radiated ultraviolet (UV) light is proportional to the total energy deposited. However, it is highly sensitive to the assumptions made in determining the fraction of light scattered out of the shower toward the detector and attenuated on the path to the detector. This necessitates an extensive system of instruments to monitor the properties of the troposphere above the observatory.

The vertical description of pressure, temperature, and humidity (as well as derived state variables such as air density and atmospheric depth) is essential for accurate calculation of the Rayleigh (molecular) attenuation length and the air fluorescence yield. Although molecular scattering is the dominating attenuation effect, we must also consider scattering due to aerosol particles. Vertical aerosol distributions can change significantly from hour to hour, as well as throughout the year.

Clouds may partly obscure the FD view of the shower, causing a break in the shower light profile and an underestimate of the energy, or they may lie directly in the path of the shower development, increasing the fraction of Cherenkov light scattered towards the FD and causing an overestimate of the energy. Thin cloud can also subtly distort the shape of the shower light profile.

\section{Instruments and data processing}

\subsection{Molecular monitoring}

From 2002 until the end of 2008, radiosondes were intermittently launched from the observatory to measure important air properties as a function of height and produce vertical profiles [2].

The Global Data Assimilation System (GDAS), provided by the United States National Center for Environmental Prediction, is a freely-available source of 3-hourly molecular state parameters produced by a model combining data from radiosondes, surface observations, radar observations, and satellite observations. In 2011 these vertical profiles replaced local radiosonde data in the event reconstruction for all air showers seen by the Auger Observatory since 2005 [3].

The observatory additionally uses five ground-based weather stations - one at each of the four FD buildings and one at the CLF (see Sec. 2.2) - to monitor the ground-level pressure, temperature, humidity, and wind velocity with a time resolution of $5 \mathrm{~min}$.

\subsection{Aerosol monitoring}

The Auger Observatory operates two laser facilities within the surface array, both relatively near the centre. The Central Laser Facility (CLF) has been operational since the commissioning 
of the first FD site, while the eXtreme Laser Facility was constructed later to benefit the FD sites furthest from the CLF. Each facility houses a UV laser which fires 50 vertical pulses every $15 \mathrm{~min}$. Each pulse has a width of $7 \mathrm{~ns}$ and the average pulse energy is $6.5 \mathrm{~mJ}$. Each of the two lasers are observed from each of the four FD sites, creating a bistatic lidar system of eight laser-FD pairs. For each laser-FD pair, the average of the 200 pulses in each hour creates a trace of photons at the detector as a function of height (hourly light profile).

Since 2013 there has also been a monostatic Raman lidar system at the CLF [4], used to make three measurements of the vertical aerosol profile each night: before, during, and after the FD observations. The Raman lidar cannot be operated while the FD is running, but this only interrupts data acquisition for four of the 27 fluorescence telescopes and only for 20 min each night.

In order to calculate vertical aerosol distributions with the bistatic lidar, we normalise our data periodically with "reference" nights, typically one for each year, for which the aerosol attenuation is negligible. To find a reference night we select candidates with hourly light profiles which match closely to profiles simulated under aerosol-free conditions, and cross-reference to other sources such as the Raman lidar to determine the clearest night. The data-normalised method for aerosol analysis at the Auger Observatory [5] uses the reference light profile to cancel out all detector systematics and molecular scattering effects in other light profiles each year, leaving just aerosols to explain any difference in magnitude and shape.

\subsection{Cloud monitoring}

At each FD site is a monostatic elastic backscatter lidar which automatically scans for cloud outside of the field of view (FOV) of the FD every 15 min [1]. The time delay and magnitude of the returned signal indicates the distance and density of scattering centres. In Fig. 1a an overhead FD lidar scan indicates a localised cloud base height $(\mathrm{CBH})$ of $4 \mathrm{~km}$ above ground level. During event reconstruction, the minimum $\mathrm{CBH}$ and the overhead cloud coverage $(0 \%$ to $100 \%)$ are considered.

The bistatic lidar system described above, though primarily intended for aerosol monitoring, will detect cloud when it lies either directly over the lasers or along a path between the lasers and an FD site. In the first case this provides a measurement of the $\mathrm{CBH}$ over the surface array, and in the second case an upper limit which we can still use. The overall CBH for an hour is taken as the lowest seen by any laser-FD pair in any 15 min block of laser shots.

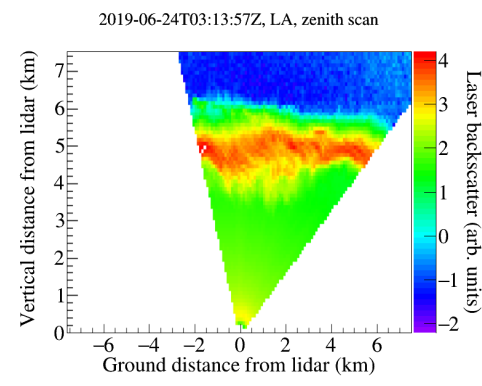

(a) FD lidar scan.

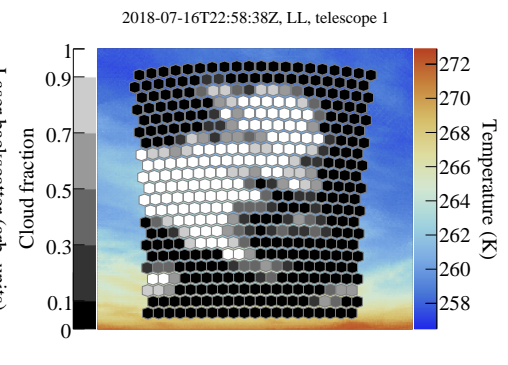

(b) Cloud camera image.

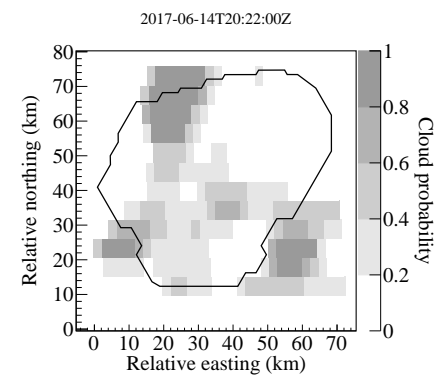

(c) Satellite cloud probability map.

Figure 1: Examples of real data from an FD lidar, a cloud camera (masked to the pixels of one fluorescence telescope), and GOES. 
Atop each FD building is an infrared (IR) camera on a motorised mount, sensitive to the band from $8 \mu \mathrm{m}$ to $14 \mu \mathrm{m}$ where clouds appear warmer than clear sky. Every 5 min the cameras scan the FOV of the FD sites, and every 15 min they scan the full sky. An example cloud camera image for one fluorescence telescope, with cloud in the FOV, is shown in Fig. 1b. The images are analysed and a cloud fraction $(0 \%$ to $100 \%)$ is associated with each FD pixel. Near the horizon (elevation $\leqslant 5.5^{\circ}$ ) the atmosphere becomes optically thick in this IR band and the higher radiative temperature of water vapour makes discerning clouds very difficult, so these cloud fractions are considered less reliable. During data acquisition, the cloud camera images are used by FD operators for guidance on outside conditions. During event reconstruction, the cloud fraction of each FD pixel along the shower track is considered.

The Geostationary Operational Environmental Satellite system (GOES) is operated by the United States National Oceanic and Atmospheric Administration. The raw data are freely available, and an algorithm tuned for use at the Auger Observatory is applied to convert aerial measurements across four IR bands into cloud probability maps every $30 \mathrm{~min}$. Fig. 1c is an example of such a map, which allocates a probability of cloud ( $0 \%$ to $100 \%$, in $20 \%$ steps) to each $2.4 \mathrm{~km} \times 5.5 \mathrm{~km}$ satellite imagery pixel across the surface array [6]. During event reconstruction, the cloud probability of each satellite pixel between the FD and the shower axis from above is considered.

\section{Application of data during air shower analysis}

\subsection{Molecular data}

The atmospheric density profile plays a fundamental role in converting the light emitted at the shower track as a function of height, to the energy deposited in the atmosphere per unit depth as a function of depth. It is also used to account for molecular scattering and attenuation when converting the observed fluorescence light into energy deposited at the shower.

The pressure, temperature, and humidity profiles are used to calculate the fluorescence yield along the track of the air shower. This includes accounting for the temperature dependence of the collisional cross-sections of atmospheric nitrogen with nitrogen or oxygen, the frequency of collisions, and additional de-excitation by water vapour [3].

Variations in pressure and air density close to the ground affect the signal observed in the SD due to changes in particle scattering. This modulates the reconstructed shower energy, and for studies using a fixed energy cut the diurnally modulated arrival rate can appear as a large scale anisotropy. Hence, the real-time estimations of these variables are used to apply a "weather correction" [7].

\subsection{Aerosol data}

A quality cut is placed on the value of vertical aerosol optical depth at $4.5 \mathrm{~km}$ ASL. Events are discarded from analysis when this value exceeds 0.1 , which corresponds to a $\sim 10 \%$ reduction in vertical light transmission. As indicated in Fig. 2, this is a significant minority of all events.

For studies needing high quality FD observations, we require that real aerosol data be available from the time the event occurred. We have extended the aerosol database with two more years of measurements, up to the end of 2017, allowing a larger sample of FD events to meet this requirement and potentially be used in analysis. 
Important corrections to the datanormalised method for aerosol analysis were previously described by the Auger Collaboration [8]. The effect of these changes was to remove the assumptions that aerosol scattering out of the laser beam is negligible and that multiple scattering of photons en route to the detector is negligible. These corrections were effectively incorporated to the existing software, however they could not be fully realised until the software used laser light profiles calculated with the same technique of light integration across the FD cameras as that employed for air shower reconstructions. This represented a fundamental

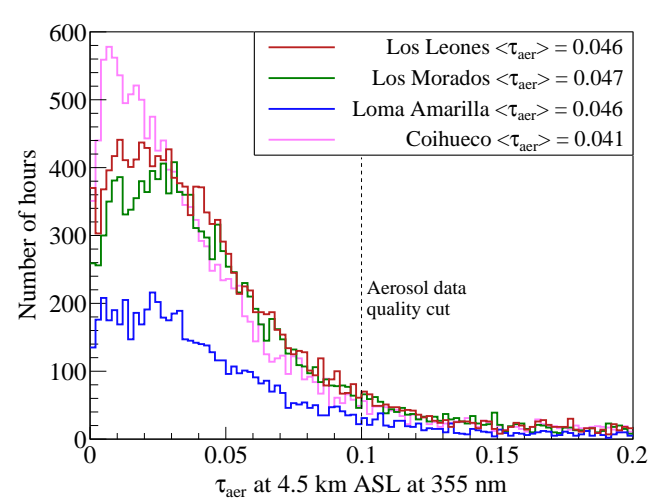

Figure 2: Distributions of vertical aerosol optical depth $\left(\tau_{\text {aer }}\right)$ at $4.5 \mathrm{~km}$ ASL for each FD site. Loma Amarilla has six fewer years of data. change, and ultimately it could only be realised after a complete rewrite of the software. This work has been completed, and as a benefit several other improvements have been made.

At very low altitudes, the integrated optical depth remains the same but we have improved the determination of the exact shape. At higher altitudes we now more rigorously apply a smoothing algorithm that prevents statistical fluctuations in the aerosol profile being interpreted as a significant step in aerosol content. We also reviewed the technique used to propagate uncertainties from the initial light profiles to the final aerosol profile, and replaced a numerical method with a much faster and more accurate analytical one. The software now has a modular structure, and any future corrections will be easier to incorporate.

All new results have been cross-checked with the separate laser simulation technique [5], finding good agreement. The new software produced the complete aerosol database used in air shower reconstructions referred to throughout Auger Collaboration contributions to these proceedings.

\subsection{Cloud data}

Data from all cloud monitoring instruments are brought together during analysis to determine if an event as viewed from a particular FD site is affected by cloud and should be discarded. These "cloud cuts" have been briefly described previously [9], but we shall now discuss them in detail.

As illustrated by Fig. 3, we test the instruments in a preferred order to determine if any indicate the event is not affected by cloud. Only when all available instruments suggest the event is cloudaffected do we conclude this is the case.

In the following list we refer to the "best approximation" for the cloud base height $(\mathrm{CBH})$ or cloud coverage from FD lidars. The best approximation is defined as the sole measurement from the FD lidar at the same FD site as viewed the event, or if unavailable then it is the average of the measurement from all other FD lidars. With reference to the labelled steps in Fig. 3:

(a) If all FD pixels which received light from the shower axis have cloud fractions (from cloud camera) of $0 \%$, then the event is clear. Cloud fractions are ignored for any FD pixels that view part of the shower below $5.5^{\circ}$ elevation, due to cloud camera limitations (see Sec. 2.3). 


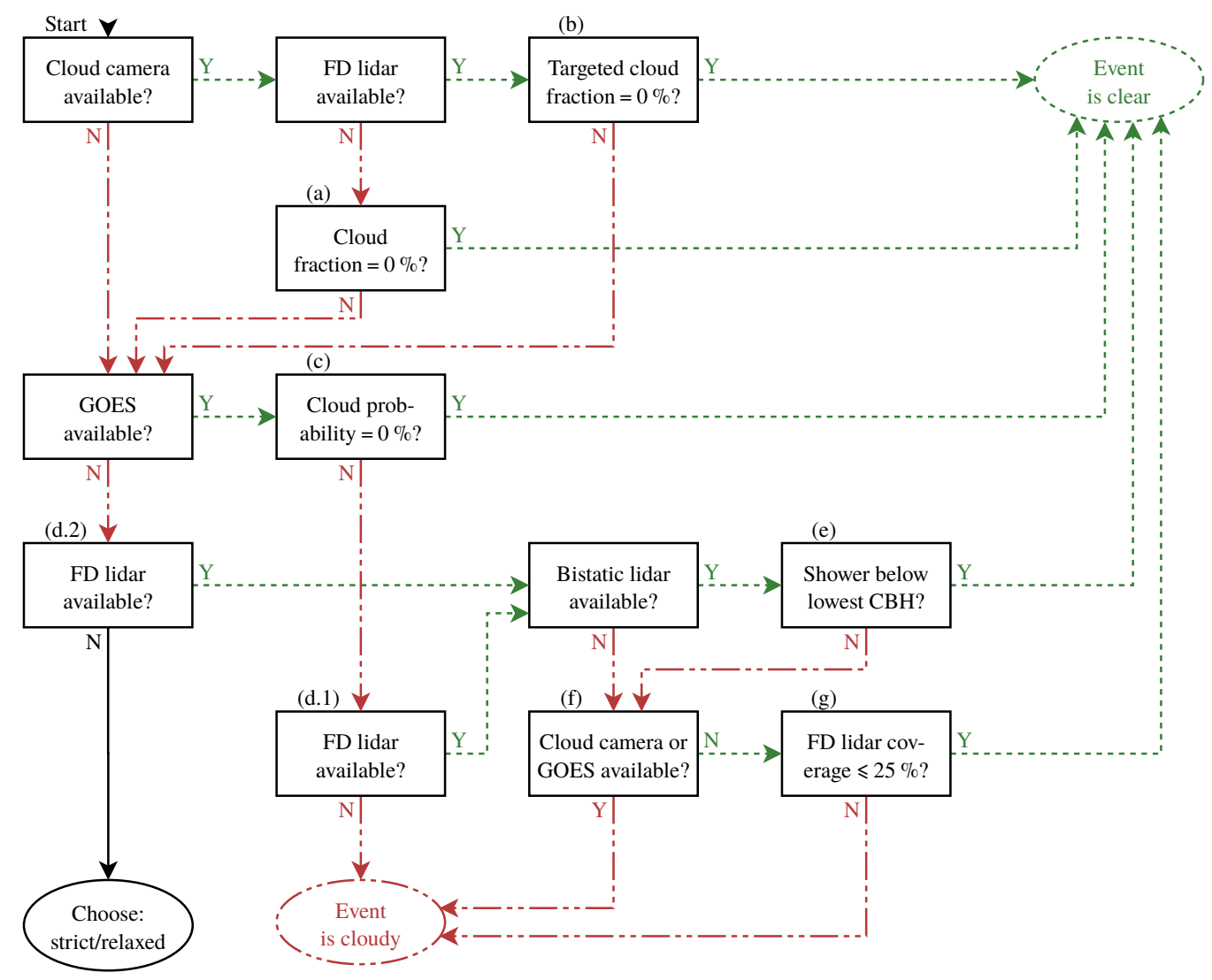

Figure 3: The cloud cut procedure for event analysis. Boxes represent steps of the procedure and ellipses represent exit points. Each step poses a question which may be answered "yes" (Y) or "no" (N). Refer to the text for detail on the steps labelled (a) through (g).

(b) As step (a), but also disregarding cloud fractions for any FD pixels viewing parts of the shower below the $\mathrm{CBH}$ of the nearest available FD lidar, because such cloud must actually be behind the shower and couldn't have affected it.

(c) If all satellite pixels between the FD site and the observed points along the shower axis have cloud probabilities of $0 \%$, then the event is clear.

(d) We cannot attempt any further tests without FD lidar, so if it is unavailable:

(d.1) Step (c) indicated cloud, so we conclude the event is cloudy.

(d.2) We cannot make a definitive conclusion and we may choose to discard or retain the event for a "strict" or "relaxed" cut respectively. The relaxed cut is generally used, but this choice depends on the specific analysis being performed.

(e) Consider the lower of the bistatic lidar CBH (see Sec. 2.3) and the best approximation of the FD lidar $\mathrm{CBH}$. If the field of view of the event observation is below this $\mathrm{CBH}$, as determined by two metrics beyond the scope of this discussion [9], then the event is clear.

(f) If cloud camera or GOES are available, then steps (b) or (c) indicated the event is cloudy.

(g) If the best approximation of the FD lidar cloud coverage is less than $25 \%$, then the event is clear. Otherwise the event is cloudy.

Even if an event affected by cloud mistakenly passes the cloud cuts, it can still be removed by the 
independent profile quality cuts. These cuts ensure that there are no large gaps in the shower light profile, that it isn't too short, and that the goodness of fit parameter is acceptable [9].

\section{Effect on reconstruction results}

\subsection{Molecular atmosphere}

Using GDAS 3-hourly data in air shower reconstructions brings improvements over the models initially employed at the observatory, which were derived from radiosonde data.

The exact effect on uncertainties was assessed by simulating events using known atmospheric profiles collected with radiosondes, reconstructing once with the old models and once with GDAS. The statistical uncertainty on energy measurements due to the molecular atmosphere is reduced by up to $50 \%$ at high energies, and for depth of shower maximum it is halved at all energies [3].

Applying the weather correction rescales the SD signal - and hence the reconstructed energy by up to $2 \%$ for most events and up to $7 \%$ in extreme cases, with some zenith angle dependence [7]. It also accounts for modulation in the daily and hourly event rates above a fixed energy threshold.

\subsection{Aerosol atmosphere}

To assess the importance of hourly aerosol measurements, we have studied the effect of using a fixed average vertical distribution of aerosols on measurements of energy $(E)$ and depth of shower maximum $\left(X_{\max }\right)$. A similar study has been done in the past [10], but here we update that work with greater statistics and a more recent aerosol analysis. We consider two average vertical aerosol profiles, one for the three FD sites at $1.4 \mathrm{~km}$ ASL and one for the site at $1.7 \mathrm{~km} \mathrm{ASL}$. We reconstruct well-measured air showers twice, once each with the average and hourly aerosol profiles, and produce the plots in Fig. 4 showing the changes in reconstructed $E$ and $X_{\max }$ and their energydependence when using these different sources of aerosol information.

These distributions exhibit large non-Gaussian tails, and an increasing standard deviation with increasing shower energy. This is a consequence of the average shower distance increasing with energy, and the hourly aerosol measurements being increasingly important for those showers. We note that the current statistical uncertainty on FD measurements of energy is $8 \%$ [11], and from $15 \mathrm{~g} \mathrm{~cm}^{-2}$ to $25 \mathrm{~g} \mathrm{~cm}^{-2}$ for depth of shower maximum [9], using hourly aerosol profiles. The use of an average profile instead would significantly worsen these resolutions at high energies, and would complicate analysis given the non-Gaussian tails.

\section{Conclusion}

An extensive range of instruments is used to monitor the state of the atmosphere at the Auger Observatory, of which a subset have been outlined here. We have discussed exactly how these instruments are used in analysis, detailing both the recent upgrades to the aerosol software as well as the full procedure for selecting and removing cloud-affected events. Finally, we have shown the effects that using real-time molecular and aerosol data have on air shower reconstructions, contrasting each against the use of average models. It is clear that the real-time aspect of these monitoring systems is a significant advantage to the science results of the Auger Observatory. 

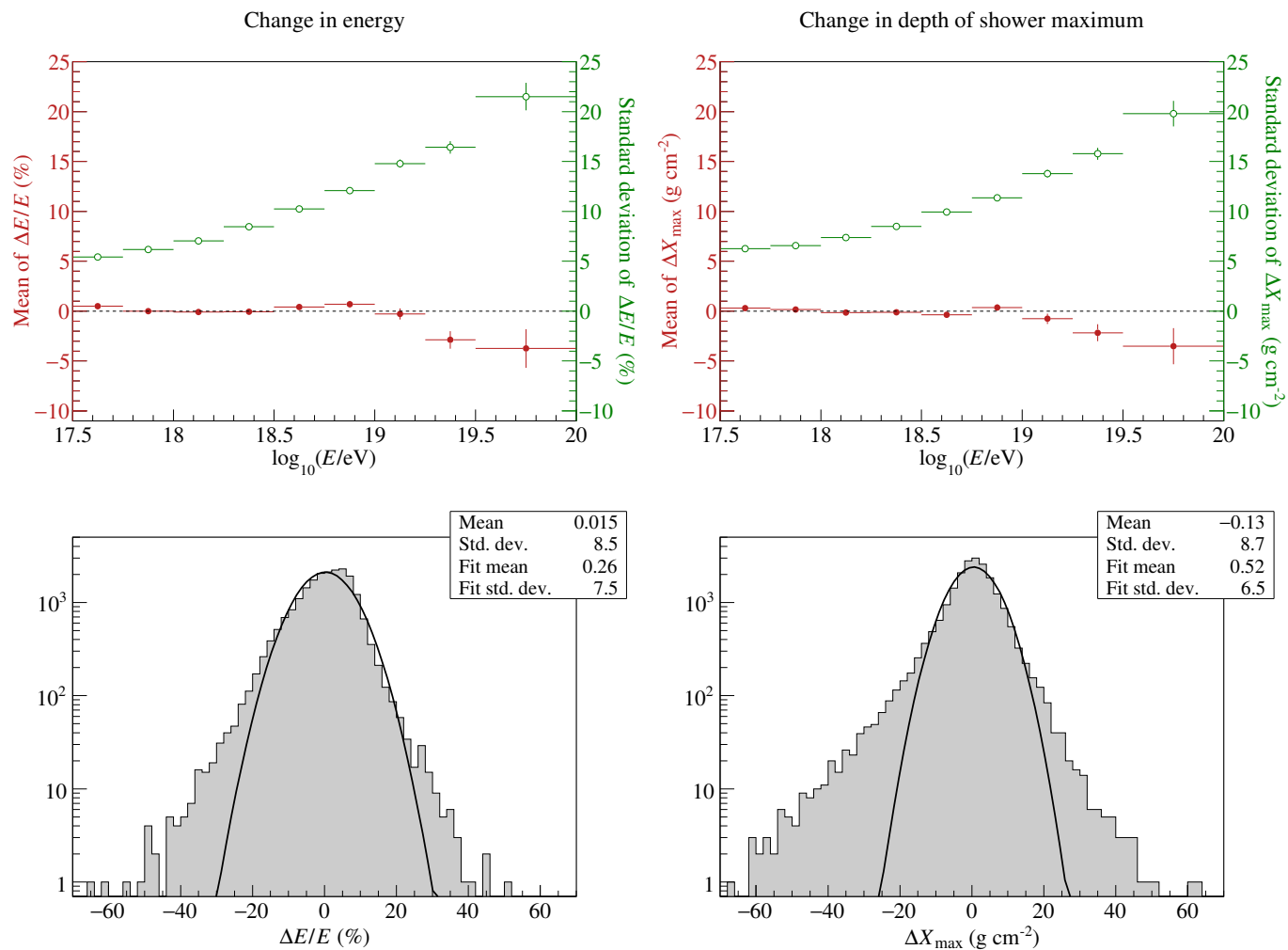

Figure 4: Results from air shower reconstructions using an average aerosol profile minus those from an hourly profile. Top: shifts in the reconstruction of FD energy $(E)$ and depth of shower maximum $\left(X_{\max }\right)$ as a function of the shower energy. Filled red circles plot the mean of the distribution in each energy bin, and open green circles plot the standard deviation. Bottom: distributions of the shifts in $E$ and $X_{\max }$ combining all energy bins, plotted with Gaussian fits.

\section{References}

[1] A. Aab et al. (Pierre Auger Collaboration), Nucl. Instrum. Methods Phys. Res., Sect. A 798, 172 (2015).

[2] P. Abreu et al. (Pierre Auger Collaboration), J. Instrum. 7, P09001 (2012).

[3] P. Abreu et al. (Pierre Auger Collaboration), Astropart. Phys. 35, 591 (2012).

[4] V. Rizi et al. (for the Pierre Auger Collaboration), EPJ Web Conf. 197, 02003 (2019).

[5] P. Abreu et al. (Pierre Auger Collaboration), J. Instrum. 8, P04009 (2013).

[6] P. Abreu et al. (Pierre Auger Collaboration), Astropart. Phys. 50-52, 92 (2013).

[7] A. Aab et al. (Pierre Auger Collaboration), J. Instrum. 12, P02006 (2017).

[8] M. Malacari (for the Pierre Auger Collaboration), Proc. Int. Cosm. Ray Conf., PoS(ICRC2017) 398.

[9] A. Aab et al. (Pierre Auger Collaboration), Phys. Rev. D 90, 122005 (2014).

[10] J. Abraham et al. (Pierre Auger Collaboration), Astropart. Phys. 33, 108 (2010).

[11] B. Dawson (for the Pierre Auger Collaboration), Proc. Int. Cosm. Ray Conf., PoS (ICRC2019) 231. 